INPLASY

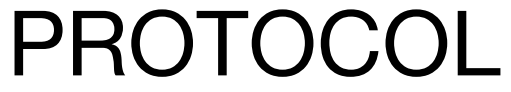

To cite: Li et al. Effects of Core

Strength Training on Martial arts Performance: a

Systematic Review. Inplasy protocol 202220108. doi:

10.37766/inplasy2022.2.0108

Received: 24 February 2022

Published: 24 February 2022

Corresponding author:

Li Long

980574879@qq.com

Author Affiliation:

College of Education,

University Putra Malaysia.

Support: At his own expense.

Review Stage at time of this submission: Data analysis.

Conflicts of interest:

None declared.

\section{Effects of Core Strength Training on Martial arts Performance: a Systematic Review}

Li, L'; Soh, KG²; Li, H³; Othman, T4; Mohamad, THbB5; Soh, KL6

Review question / Objective: Population Martial arts students (male/female), must be more then 10 subjects; Intervention Core strength, training for more than 4 weeks; Comparison Two or more groups and single-group trials; Outcome (i) Taekwondo (Basic physical qualities speed quality and Balance); (ii) karate (physical qualities); (iii)Muay Thai (Impact force and Strike velocity quality).

Eligibility criteria: 1. Participants have training in martial arts. 2. The dependent variable was core strength, while the independent variable was the performance of martial arts (Basic physical qualities speed quality and Balance Impact force and Strike velocity quality).3. Interventions were experimental in nature.4. The study has data for one or more of the following parameters: attack, body stability, movement balance, drill level, and movement difficulty.5. The study has been published in full in a peer-reviewed journal.

INPLASY registration number: This protocol was registered with the International Platform of Registered Systematic Review and Meta-Analysis Protocols (INPLASY) on 24 February 2022 and was last updated on 24 February 2022 (registration number INPLASY202220108).

\section{INTRODUCTION}

Review question / Objective: Population Martial arts students (male/female), must be more then 10 subjects; Intervention Core strength, training for more than 4 weeks; Comparison Two or more groups and single-group trials; Outcome (i) Taekwondo (Basic physical qualities speed quality and Balance); (ii) karate (physical qualities); (iii)Muay Thai (Impact force and Strike velocity quality).

Rationale: In December, 2021, a systematic literature search was conducted on the PubMed, and Scopus databases. This search reviewed the bibliography of qualified articles and related reviews. 
Randomized controlled trials and singlegroup trials were included to evaluate the effects of core strength training on Martial arts performances, and the qualifying reviews were synthesized according to PRISMA guidelines. A panel of three authors evaluated the quality of the study.

Condition being studied: Background: Core strength training is a world recognized training method, which can effectively improve the physical quality of athletes, so it is used in many sports. For example, basketball, volleyball, training. However, its impact on Martial arts performance is not clear. Objective: A systematic literature review was conducted to analyze the effects of core strength training on the performance of Martial arts practitioners. Method: In December, 2021, a systematic literature search was conducted on the PubMed, and Scopus databases. This search reviewed the bibliography of qualified articles and related reviews. Randomized controlled trials and singlegroup trials were included to evaluate the effects of core strength training on Martial arts performances, and the qualifying reviews were synthesized according to PRISMA guidelines. A panel of three authors evaluated the quality of the study. Results: From the 68 studies shortlisted, 10 were found to meet the inclusion criteria, and their findings on the influences of core strength training on the following martial arts performances were studied in detail: Taekwondo ( $n=5)$, karate $(n=4)$ Muay Thai $(n=1)$. All these studies report that core strength is beneficial and contributes to improved performances in martial arts players. Conclusion: Core strength training is helpful to the kicking speed, body balance and leg strength of Taekwondo athletes, and improves the rotation and round kick of karate athletes, boxing speed and hitting ability of Muay Thai athletes.

\section{METHODS}

Search strategy: The search for relevant literature studying the effects of core strength training on martial arts performance was conducted on English databases, such as PubMed, Scopus, and EBSCOHOST. We entered keywords and synonyms for the searches as follows: "Core strength training" OR "Core-Strength Exercise" OR "Core-Exercise" OR "Core Strength" OR "Core-Stability Exercise" OR "Core-Stability Training" OR "Taekwondo" OR "karate" "Muay Thai".

Participant or population: Taekwondo athlete, Muay Thai athlete, karate athlete.

Intervention: The influence of core strength training on taekwondo athletes, Muay Thai athletes and karate athletes.

Comparator: Two or more groups and single-group trials.

Study designs to be included: PICO was used as the basis for inclusion criteria in this paper. The study looked at whether the following criteria was met in the literature being considered for review: (1) the participants must have been members of the Taekwondo and karate profession, regardless of occupation, age, or gender; (2) the paper included an experimental study on the intervention of core strength training with the primary goal of improving the performance level of Taekwondo karate and Muay Thai practitioners, and the intervention period must have been for at least four weeks; (3) no less than 10 participants were.

Eligibility criteria: 1. Participants have training in martial arts. 2. The dependent variable was core strength, while the independent variable was the performance of martial arts (Basic physical qualities speed quality and Balance Impact force and Strike velocity quality).3. Interventions were experimental in nature.4. The study has data for one or more of the following parameters: attack, body stability, movement balance, drill level, and movement difficulty.5. The study has been published in full in a peer-reviewed journal.

Information sources: The search for relevant literature studying the effects of core strength training on martial arts 
performance was conducted on English databases, such as PubMed, Scopus, and EBSCOHOST. After the article retrieval system computed the searched keywords, analysis was done on the suggested articles' titles. The titles of the articles produced were checked to see if they included the terms 'core strength training', 'Taekwondo performance', 'karate performance, and Muay Thai performance'. Then the abstracts of the selected.

Main outcome(s): Study; Year \& location; Study type(Methods); Sample size; Intervention; Session duration/ Length of intervention; Participant details (sex); Outcome; A total of Four hundred sixtythree participants were included in these studies. The target population of this review is diverse, including five Taekwondo athletes, four karate athletes, and Thai athletes. The features of the interventions used by the 10 included pieces of literature can be categorized into the following categories: (1) the intervention period was between 8-24 weeks, and the intervention lasted for 5 weeks with two items ; (2) the intervention lasted for 8 weeks with two items ; (3) the intervention lasted for 12 weeks with two items ; (4) the intervention lasted for 16 weeks with two items ; and (5) the intervention lasted for 24 weeks with one item. Thus, this systematic review was based on 10 studies which all focused on the skills of athletes in various types of martial arts.

Quality assessment / Risk of bias analysis: The scheme of this systematic review was developed according to the Preferred Systematic Review and Meta-Analysis (PRISMA) guidelines.

Strategy of data synthesis: In order to identify relevant studies in the first screening stage, two independent reviewers (Li Long and $\mathrm{Li} \mathrm{Hu}$ ) screened the titles and abstracts of all the identified literature. After evaluating the titles and abstracts based on the predetermined inclusion and exclusion criteria, irrelevant papers were removed from the database. The full text was evaluated if the title and summary did not provide enough information as to whether the paper met the criteria. The full texts of the potentially qualifying literature were reviewed in the second screening stage. If a disagreement arose regarding the qualification of a study, a third reviewer (Xiao) would be consulted to reach a consensus. After evaluating the complete texts, 10 pieces of literature met our inclusion criteria and were included in the systematic evaluation.

Subgroup analysis: 3.3.1 Effect of Core strength training on the Performance of Taekwondo (i) Basic physical qualities (ii) The speed quality (iii) Balance quality 3.3.2 Effect of Core strength training on karate Performance physical qualities 3.3.3 Effect of Core strength training on Muay Thai Performance (i)Impact force (ii)Strike velocity.

Sensitivity analysis: The results of this study focus on the influence of Core strength training on the different performances of martial arts. It summarizes all existing literature on the effects of Core strength training on martial arts performances. The results of the review found that the intervention used in a study has varying effects on the different categories of martial arts. These effects can be categorized into: 1. Effect of Core strength training on the performance of Taekwondo (Basic physical qualities speed quality and Balance). 2. Effect of Core strength training on karate (physical qualities) 3. Effect of Core strength training on the performance of Muay Thai (Impact force and Strike velocity quality).

\section{Language: English and Chinese.}

Country(ies) involved: Malaysia and China.

Keywords: Core strength training; Martial arts; Taekwondo; karate; MuayThai.

Contributions of each author:

Author 1 - Li Long - Conceived this review. Email: 980574879@qq.com

Author 2 - Soh Kim Geok - Drafted the protocol under the supervision.

Email: kims@upm.edu.my 
Author 3 - Li Hu - Reviews the collected data.

Email: 544126171@qq.com

Author 4 - Othman Talib - Conducted data collection.

Email: 332166460@qq.com

Author 5 - Taufik Hidayat bin Baharuldin -

Conducted data collection.

Email: taufik@upm.edu.my

Author 6 - Soh Kim Lam - Conducted data

collection.

Email: soh@upm.edu.my 